Bull. Korean Math. Soc. 49 (2012), No. 5, pp. 939-947

http://dx.doi.org/10.4134/BKMS.2012.49.5.939

\title{
EMBEDDING RIEMANNIAN MANIFOLDS VIA THEIR EIGENFUNCTIONS AND THEIR HEAT KERNEL
}

\author{
Hiba AbDallah
}

\begin{abstract}
In this paper, we give a generalization of the embeddings of Riemannian manifolds via their heat kernel and via a finite number of eigenfunctions. More precisely, we embed a family of Riemannian manifolds endowed with a time-dependent metric analytic in time into a Hilbert space via a finite number of eigenfunctions of the corresponding Laplacian. If furthermore the volume form on the manifold is constant with time, then we can construct an embedding with a complete eigenfunctions basis.
\end{abstract}

\section{Introduction}

Let $(M, g)$ be a Riemannian manifold. The diffusion operator related to the Laplacian is the heat operator, and it is an infinitely smoothing operator. It admits a fundamental solution of class $C^{\infty}$ which is called the heat kernel. If we denote this kernel by $K(t, x, y), t>0, x, y \in M$, then it verifies the following equation:

$$
\left(\Delta_{x}-\frac{\partial}{\partial t}\right) K(t, x, y)=0
$$

with initial data

$$
\lim _{t \rightarrow 0} K(t, x, y) d x=\delta_{y}(x)
$$

for each $y \in M$, e.g. for any continuous function $f$ on $M$ with compact support, we have

$$
\lim _{t \rightarrow 0} \int_{M} K(t, x, y) f(x) d v(x)=f(y) .
$$

More precisely, let $(M, g)$ be a compact Riemannian manifold without boundary, $\Delta$ the Laplace-Beltrami operator on $M$, and let $\lambda_{0}<\lambda_{1} \leq \lambda_{2} \leq \lambda_{3} \cdots \nearrow \infty$

Received May 9, 2011; Revised September 13, 2011.

2010 Mathematics Subject Classification. 47B40, 53B21, 54A20, 58A32, 58C25, 58C40.

Key words and phrases. Riemannian manifold, Laplacian, eigenvalues/eigenfunctions, heat equation, embedding. 
denote the eigenvalues of $\Delta$, and $\left\{\varphi_{i}\right\}_{i \geq 0}$ an orthonormal basis of real eigenfunctions of $\Delta$. Then the heat kernel of $M$ can be expressed as follows:

$$
K(t, x, y)=\sum_{i \in \mathbb{N}} e^{-\lambda_{i} t} \varphi_{i}(x) \varphi_{i}(y), \quad t>0, x, y \in M
$$

The diffusion distance on $(M, g)$ is defined by

$$
D_{t}^{2}(x, y)=\int_{M}(K(t, x, z)-K(t, y, z))^{2} d v(z) .
$$

Although the notions "diffusion distance" have been introduced by Coiffman and Lafon, in 2004, in their paper [4], this notion was not really new. In fact, in [3], P. Bérard, G. Besson, and S. Gallot, used the heat kernel to embed a class of closed Riemannian manifolds in the same Hilbert space

$$
l^{2}=\left\{\left(a_{j}\right)_{j \geq 1}, a_{j} \in \mathbb{R} ; \sum_{j \geq 1}\left|a_{j}\right|^{2}<+\infty\right\},
$$

and they interpret certain estimates on the heat kernel as giving a precompactness theorem on the considered class. We have the following:

Theorem 1.1 (P. Bérard, G. Besson and S. Gallot [3]). Let $(M, g)$ be an $n$ dimensional closed Riemannian manifold $(M, g)$ and $\lambda_{0}<\lambda_{1} \leq \lambda_{2} \leq \cdots \nearrow \varnothing$ the eigenvalues of the correspondent Laplacian $\Delta_{M}$. The map

$$
\begin{gathered}
\psi_{t}^{a}: M \rightarrow l^{2} \\
x \mapsto \sqrt{2}(4 \pi)^{\frac{n}{4}} t^{(n+2) / 4}\left\{e^{-\lambda_{j} t / 2} \varphi_{j}^{a}(x)\right\}_{j \geq 1}
\end{gathered}
$$

is an embedding for each $t>0$, and each orthonormal basis $a=\left\{\varphi_{i}^{a}\right\}_{\{i \geq 0\}}$ of real eigenfunctions of $\Delta_{M}$. Moreover, the pull-back metric $\left(\psi_{t}^{a}\right)^{*}$ can, where "can" denote the Euclidian scalar product on $l^{2}$, is asymptotic to the metric $g$ of $M$ when $t$ goes to zero. More precisely,

$$
\left(\psi_{t}^{a}\right)^{*} \text { can }=g+\frac{t}{3}\left(\frac{1}{2} S c a l_{g} . g-R i c_{g}\right)+O\left(t^{2}\right), \text { when } t \rightarrow 0_{+}
$$

where $\mathrm{Scal}_{g}$ and Ric $\mathrm{C}_{g}$ are respectively the scalar curvature and the Ricci curvature tensor of the metric $g$.

The map $\psi_{t}^{a}$ can be called "diffusion map" (see [4]), and the distance in $l^{2}$ between the images $\psi_{t}^{a}(x), \psi_{t}^{a}(y)$ of two points $x, y \in M$ is equal to the diffusion distance defined by the equation (3) (up to a constant that serves here for normalization).

The proof of Theorem 1.1 lies on the fact that the heat kernel can be written in terms of all the laplacian eigenfunctions and eigenvalues (see Equation 2). However, we can also embed a Riemannian manifold via a finite number of eigenfunctions. And we have the following theorem: 
Theorem 1.2 (P. Bérard [2]). The map

$$
\begin{aligned}
\Phi_{\lambda}: M & \rightarrow \mathbb{R}^{N(\lambda)+1} \\
x & \mapsto\left(\frac{\varphi_{0}(x)}{\nu(x, \lambda)}, \ldots, \frac{\varphi_{N(\lambda)}(x)}{\nu(x, \lambda)}\right)
\end{aligned}
$$

is an embedding for $\lambda$ large enough, and, $\Phi_{\lambda}(M) \subset S^{N(\lambda)}(1)$ (sphere of radius 1 in $\mathbb{R}^{N(\lambda)+1}$ ), and for all $V \in T M \backslash\{0\}$,

$$
\left\|d \Phi_{\lambda}(V)\right\|_{\mathbb{R}^{N(\lambda)+1}}^{2}=\frac{\lambda}{n+2}\left[g_{0}(V, V)+b(V, \lambda)\right],
$$

where $b(V, \lambda)=o(1)$ uniformly in $V$ in the unit tangent bundle UTM when $\lambda$ approaches to $+\infty$.

The aim of this paper is to generalize the results above to the case where $M$ is endowed by a time-dependent metric. So we consider the flow $(M, g(t))$ where $g(t)$ is analytic in $t \in]-T, T$. We prove the existence of a finite number of eigenfunctions of $\Delta_{g(t)}$, all analytic in $t$ in a neighborhood of $t=0$, and we construct an embedding by these eigenfunctions. We have our first result that is the following:

Theorem 1.3. Let $(M, g(t))$ be a family of Riemannian manifolds such that $g(t)$ is analytic in $t \in]-T, T[, T>0$.

i) There exist $\varepsilon_{0}>0, N_{0} \in \mathbb{N}$, such that, for all $N \geq N_{0}$, there exist $\epsilon_{N}>0, N+1$ eigenfunctions $\varphi_{i}(t)$ of $\Delta_{g(t)}, i=0, \ldots, N$, all analytic in $t$ for $t \in]-\epsilon_{N}, \epsilon_{N}[$, such that the map

$$
\begin{aligned}
\Phi_{t}^{N}:(M, g(t)) \rightarrow \mathbb{R}^{N+1} \\
x \mapsto \frac{2(4 \pi)^{\frac{n}{2}} \Gamma\left(\frac{n}{2}+2\right)}{\left(\lambda_{N}(t)\right)^{\frac{n}{2}+1}}\left(\varphi_{0}(t, x), \ldots, \varphi_{N}(t, x)\right)
\end{aligned}
$$

is an embedding for all $|t|<\min \left\{\varepsilon_{0}, \epsilon_{N}\right\}$ (here $\Gamma$ is the well-known Gamma function).

ii) The pull-back metric of $\Phi_{t}^{N}$ is asymptotic to the metric $g(t)$ of $(M, g(t))$ when $N$ goes to infinity. More precisely

$$
\left(\Phi_{t}^{N}\right)^{*} \text { Can }=g(t)\left(1+O\left(\frac{1}{\ln \left(\lambda_{N}(t)\right)}\right)\right) \text {, when } N \rightarrow \infty
$$

where Can is the Euclidian metric of $\mathbb{R}^{N_{0}+1}$.

In the third section, we see that if the volume form on $(M, g(t))$ is constant, then there exists a complete orthonormal basis of eigenfunctions of $\Delta_{g(t)}$, all analytic in time, and we can embed the flow $(M, g(t))$ with the associated heat kernel. 


\section{Embedding a flow of Riemannian manifolds via a finite number of eigenfunctions}

Let $(M, g(t)), t \in[-T, T]$ a family of Riemannian manifolds where $g(t)$ is analytic in $t \in]-T, T$. In this section we prove Theorem 1.3, and for this purpose, we need to prove the following lemma on perturbed operators.

Lemma 2.1. If $\lambda_{0} \leq \lambda_{1} \leq \cdots \leq \lambda_{k}$, are the $k$-th first eigenvalues of $\Delta_{0}$, there exists $\epsilon_{k}>0$, and for all $i=1, \ldots, k$, there exist a scalar-valued function $\lambda_{i}(t)$, and a $C^{\infty}(M)$-valued function $\varphi_{i}(t)$, all analytic in $|t|<\epsilon_{k}$, such that

(i) $\Delta_{t} \varphi_{i}(t)=\lambda_{i}(t) \varphi_{i}(t)$ for all $|t|<\epsilon_{k}$,

(ii) $\lambda_{i}(0)=\lambda_{i}$,

(iii) $\left\{\varphi_{i}(t)\right\}_{i \leq k}$ is an orthonormal system of $L^{2}\left(M, v_{t}\right)$ for all $|t|<\epsilon_{k}$.

Proof. Let $\nu_{1}<\cdots<\nu_{p}$ be the first $p$ distinctive eigenvalues of $\Delta_{0}$ of multiplicity $m_{i}$ respectively, $i=1, \ldots, p$. Then, by P. Bérard [1] and F. Rellich [9], there exist, for all $i=1, \ldots, p, \epsilon_{i}>0, m_{i}$ scalar-functions $\lambda_{i_{l}}(t), l=1, \ldots, m_{i}$, and $m_{i}$ functions $\varphi_{i_{l}}(t)$ with values in $C^{\infty}(M)$ all analytic in $|t|<\epsilon_{i}$, and verifying

(1) $\Delta_{t} \varphi_{i_{l}}(t)=\lambda_{i_{l}}(t) \varphi_{i_{l}}(t)$ for all $|t|<\epsilon_{i}$,

(2) $\lambda_{i_{l}}(0)=\nu_{i}$

(3) $\left\{\varphi_{i_{l}}(t)\right\}_{l=1}^{m_{i}}$, is an orthonormal system of $L^{2}\left(M, v_{t}\right)$ for all $|t|<\epsilon_{i}$.

Further, since $M$ is compact, its spectrum is separated, and for all $|t|<\epsilon_{i}$, $i=1, \ldots, p$, the spectrum of $\Delta_{g(t)}$ in the interval $\left[\nu_{i}, \nu_{i+1}\right.$ [ consists of the points $\lambda_{i_{l}}(t), l=1, \ldots, m_{i}$.

We set $\epsilon_{k}=\inf _{i=1}^{p}\left\{\epsilon_{i}\right\}$, and we design by $\varphi_{1}, \ldots, \varphi_{m_{1}}$ the $\varphi_{1_{l}}$, by $\varphi_{m_{1}+1}, \ldots$, $\varphi_{m_{1}+m_{2}}$ the $\varphi_{2_{l}}$, and so on. In such wise we obtain $k$ functions $\phi_{i}(t)$ and $k$ functions $\lambda_{i}(t)$, all analytic in $t$ for $|t|<\epsilon_{k}$ such that

(i) $\Delta_{t} \varphi_{i}(t)=\lambda_{i}(t) \varphi_{i}(t)$ for all $|t|<\epsilon_{k}$,

(ii) $\lambda_{i}(0)=\lambda_{i}$,

(iii) If $\lambda_{i}=\lambda_{j}$, then by (2) of this proof $\left\langle\phi_{i}(t), \phi_{j}(t)\right\rangle_{g(t)}=\delta_{i j}$. If $\lambda_{i} \neq \lambda_{j}$, then by (4), $\lambda_{i}(t) \neq \lambda_{j}(t)$, and $\Delta_{g(t)}$ is autoadjoint,

$$
\left\langle\Delta_{g(t)} \phi_{i}(t), \phi_{j}(t)\right\rangle_{g(t)}=\left\langle\phi_{i}(t), \Delta_{g(t)} \phi_{j}(t)\right\rangle_{g(t)},
$$

e.g.

$$
\left\langle\lambda_{i}(t) \varphi_{i}(t), \varphi_{j}(t)\right\rangle_{g(t)}=\left\langle\varphi_{i}(t), \lambda_{j}(t) \varphi_{j}(t)\right\rangle_{g(t)},
$$

then $\left\langle\varphi_{i}(t), \varphi_{j}(t)\right\rangle_{g(t)}=0$, and

$\left\langle\varphi_{i}(t), \varphi_{j}(t)\right\rangle_{g(t)}=\delta_{i j}$, and $\left\|\varphi_{i}(t)\right\|_{g(t)}=1$ for all $i, j=1, \ldots, k$.

We also need the following lemma.

Lemma 2.2 (C. Morlet [8]). Let $M$ and $N$ be two differentiable manifolds of class $C^{s}$ (at least), where $s$ is a positive integer and $M$ is connected. Let $\operatorname{Hom}^{s}(M, N)$ denote the set of maps of class $C^{s}$ from $M$ into $N(0 \leq s \leq+\infty)$. 
Then the set of embeddings of $M$ into $N$ is an open set in $\operatorname{Hom}^{s}(M, N)$ with respect to the topology $C^{s}$ (this set can be obviously empty).

Now we have the basic elements to prove Theorem 1.3.

Proof of Theorem 1.3. i) On the one hand, by Theorem 1.2, there exists $N_{0} \in \mathbb{N}$ such that the map

$$
\begin{gathered}
\Phi_{0}: M \rightarrow \mathbb{R}^{N_{0}+1} \\
x \mapsto \frac{2(4 \pi)^{\frac{n}{2}} \Gamma\left(\frac{n}{2}+2\right)}{\left(\lambda_{N_{0}}\right)^{\frac{n}{2}+1}}\left(\varphi_{0}(0, x), \ldots, \varphi_{N_{0}}(0, x)\right),
\end{gathered}
$$

where $\varphi_{i}(0), i=0, \ldots, N_{0}$, are the first $N_{0}+1$ eigenfunctions $\Delta_{g(0)}$, is an embedding.

On the other hand, by Lemma 2.1, for all $N \in \mathbb{N}$, there exists $\epsilon_{N}>0, N+1$ scalar functions $\lambda_{i}(t)$ and $N+1$ functions $\varphi_{i}(t)$, all analytic in $|t|<\epsilon_{1}$, such that

$$
\Delta_{g(t)} \varphi_{i}(t)=\lambda_{i}(t) \varphi_{i}(t) \text { for all }|t|<\epsilon_{N}
$$

Furthermore, for all $|t|<\epsilon_{N},\left\{\varphi_{i}(t)\right\}_{i \leq N}$ is an orthonormal system of $L^{2}\left(M, v_{g(t)}\right)$, so the map

$$
\begin{aligned}
& \Phi_{t}^{N}:(M, g(t)) \rightarrow \mathbb{R}^{N+1} \\
x & \mapsto \frac{2(4 \pi)^{\frac{n}{2}} \Gamma\left(\frac{n}{2}+2\right)}{\left(\lambda_{N}\right)^{\frac{n}{2}+1}}\left(\varphi_{0}(t, x), \ldots, \varphi_{N}(t, x)\right),
\end{aligned}
$$

is well defined for all $|t|<\epsilon_{N}$.

Let $\Phi_{t}$ denote the map $\Phi_{t}^{N_{0}}$. Now we prove that there exists $\varepsilon_{0}>0$ such that $\Phi_{t}$ is embedding for all $t<\varepsilon_{0}$. More precisely, in the flavour of Lemma 2.2 , we will prove that

$$
\lim _{t \rightarrow 0}\left\|d_{x} \Phi_{t}(V)-d_{x} \Phi_{0}(V)\right\|_{\mathbb{R}^{N_{0}+1}}=0 \text { uniformly in } V \in U T_{x} M,
$$

where $U T_{x} M=\left\{V \in T_{x} M, g(0)(V, V)=1\right\}$.

Yet, for all $i=0, \ldots, N_{0}$, there exists $\gamma_{i}>0$ such that the function $\varphi_{i}(t)$ is given by its Taylor expansion

$$
\varphi_{i}(t)=\sum_{l=0}^{\infty} \frac{\varphi_{i}^{(l)}(t)}{l !} t^{l}, \quad|t|<\gamma_{i} .
$$

Thus, let us first prove that

$$
d_{x} \varphi_{i}(t, V)=\sum_{l=0}^{\infty} \frac{d_{x} \varphi_{i}^{(l)}(t, V)}{l !} t^{l}
$$

for all $V \in U T_{x} M$, and all $i=0, \ldots, N_{0},|t| \leq \inf \left\{\gamma_{i}, 1\right\}$.

For this purpose, we fix $i \in\left\{0, \ldots, N_{0}\right\}$, and denote by

$\exp _{x}: T_{x} M \supseteq B\left(0, \frac{\rho}{2}\right) \rightarrow B\left(x, \frac{\rho}{2}\right), \quad$ where $\rho=\inf \left\{\operatorname{inj}(M, g(t)) ; t \in\left[0, \gamma_{i}\right]\right\}$ 
the exponential map with respect to the metric $g(0)$ (in fact, exp is a local chart around $x$, see [5], Chapter 3). And for all $l \in \mathbb{N}$, we denote by $\psi_{i, l}$ the function $\frac{\varphi_{i}^{(l)}(t)}{l !}$. Then for all $l \in \mathbb{N}$, we have

$$
\left|\psi_{i, l} \circ \exp _{x}(\vartheta)-\psi_{i, l} \circ \exp _{x}(0)-d_{x} \psi_{i, l}(\vartheta)\right|=o_{l}(|\vartheta|),
$$

where $|\vartheta|$ is the norm of the vector $\vartheta$ with respect to the metric $g(0)$, and for all $l \in \mathbb{N}, o_{l}(|\vartheta|)=o(|\vartheta|)$. It follows that

$$
\left|d_{x} \psi_{i, l}(\vartheta)\right| \leq\left|\psi_{i, l} \circ \exp _{x}(\vartheta)-\psi_{i, l} \circ \exp _{x}(0)\right|+o_{l}(|\vartheta|) .
$$

Now, since for all $l \in \mathbb{N}$,

$$
\lim _{|\vartheta| \rightarrow 0}\left|\psi_{i, l} \circ \exp _{x}(\vartheta)-\psi_{i, l} \circ \exp _{x}(0)\right|=0,
$$

then there exist $C_{0}>0$ and $\xi_{0}>0$ such that for all $l \in \mathbb{N}$ and all $\vartheta \in T_{x} M$ :

$$
|\vartheta| \leq \xi_{0} \Rightarrow\left|\psi_{i, l} \circ \exp _{x}(\vartheta)-\psi_{i, l} \circ \exp _{x}(0)\right| \leq C_{0} \text {. }
$$

Furthermore, there exist $C_{1}>0$ and $\xi_{1}>0$ such that for all $l \in \mathbb{N}$,

$$
|\vartheta| \leq \xi_{1} \Rightarrow\left|o_{l}(|\vartheta|)\right| \leq C_{1} .
$$

Let us define $\xi_{2}=\inf \left\{\xi_{0}, \xi_{1}\right\}$. By the equations (6), (7), and (8), we have

$$
t^{l}\left|d_{x} \psi_{i, l}(\vartheta)\right| \leq t^{l}\left(C+C_{1}\right) \text { for all } l \in \mathbb{N},|\vartheta|<\xi_{2} .
$$

Thus, for all $|t| \leq \inf \left\{\gamma_{i}, 1\right\}$ ( $\gamma_{i}$ is given in the equation (4)), the series $\sum_{l=0}^{\infty} t^{l}\left|d_{x} \psi_{i, l}\right|$ is normally convergent in the set

$$
\left\{\vartheta \in T_{x} M ; \quad|\vartheta| \leq \xi_{2}\right\}
$$

and

$$
d_{x} \varphi_{i}(t, \vartheta)=\sum_{l=0}^{\infty} t^{l} d_{x} \psi_{i, l}(\vartheta)
$$

for all $\vartheta \in T_{x} M$ such that $|\vartheta| \leq \xi_{2}$.

Since $U T_{x} M$ is compact and the functions $d_{x} \varphi_{i}(t), d_{x} \psi_{i, l}(t)$ are linear, we have equation (5).

Finally,

$$
\lim _{t \rightarrow 0}\left\|d_{x} \varphi_{i}(t, V)-d_{x} \varphi_{i}(0, V)\right\|=\lim _{t \rightarrow 0}\left\|\sum_{l=1}^{\infty} d_{x} \psi_{i, l}(V) t^{l}\right\|=0
$$

uniformly in $V \in U T_{x} M$.

Hence, according to Lemma 2.2, there exists $\varepsilon_{0}>0$, such that for all $|t|<\varepsilon_{0}$, $\Phi_{t}=\Phi_{t}^{N_{0}}$ is an embedding from $(M, g(t))$ into $\mathbb{R}^{N_{0}+1}$. Consequently, for all $N \geq N_{0}, \phi_{t}^{N}$ is an embedding from $(M, g(t))$ into $\mathbb{R}^{N+1}$ for all $\leq|t|<$ $\min \left\{\varepsilon_{0}, \epsilon_{N}\right\}$.

ii) To prove the pull-back metric, we let

$$
0=\lambda_{0}(t) \leq \lambda_{1}(t) \leq \cdots \rightarrow \infty
$$


be all the eigenvalues of the Laplacian $\Delta_{g(t)}$, and $\left\{\varphi_{i}(t)\right\}_{i \geq 0}$ the complete associated eigenfunctions basis (we don't pretend here that the $\varphi_{i}(t)$ are continuous in $t)$. Then, for all $t$, we consider the heat kernel $K_{g(t)}(s, \cdot, \cdot)$ of $(M, g(t))$, e.g. the fundamental solution of the problem

$$
\left\{\begin{array}{c}
\left(\Delta_{g(t)}-\frac{\partial}{\partial s}\right) K_{g(t)}(s, x, y)=0 \\
\lim _{s \rightarrow 0} K_{g(t)}(s, x, y)=\delta_{x}(y)
\end{array}\right.
$$

We have the following expansion

$$
K_{g(t)}(s, x, y)=\sum_{j \geq 1} e^{-\lambda_{j}(t) s} \varphi_{i}^{2}(t, x) .
$$

Since for all $t$ fixed

$$
K_{g(t)}(s, x, y)=\frac{1}{(4 \pi s)^{\frac{n}{2}}}(1+a(s, t))
$$

where $a(s, t)=O(s)$, and by the proof of Theorem 1.1 (see [3]), we have

$$
\sum_{j \geq 1} e^{-\lambda_{j}(t) s}\left(d_{x} \varphi_{i}(t, V)\right)^{2}=\frac{g(t)(V, V)}{2(4 \pi)^{\frac{n}{2}} s^{\frac{n}{2}+1}}(1+b(t, s)),
$$

where $b(t, s)=O(s)$ for all $t$. Hence, using Karamata's Theorem (see [7]), we get

$$
\sum_{i \leq N}\left(d_{x} \varphi_{i}(t, V)\right)^{2}=\frac{\left(\lambda_{N}(t)\right)^{\frac{n}{2}+1} g(t)(V, V)}{2(4 \pi)^{\frac{n}{2}} \Gamma\left(\frac{n}{2}+2\right)}\left(1+d\left(V, \frac{1}{\ln \lambda_{N}(t)}\right)\right),
$$

where $d\left(V, \frac{1}{\ln \lambda(t)}\right)=O\left(\frac{1}{\ln \lambda(t)}\right)$ when $N \rightarrow \infty$, uniformly in $V \in U T_{x} M$, $x \in M$. And

$$
\left(\Phi_{t}\right)^{*} C a n=g(t)\left(1+O\left(\frac{1}{\ln \left(\lambda_{N}(t)\right)}\right)\right) \text { when } N \rightarrow+\infty .
$$

\section{Embedding a flow of Riemannian manifolds via a complete basis of eigenfunctions}

In this section, we embed a flow of Riemannian manifolds $(M, g(t))$ using a complete orthonormal family of eigenfunctions. Given a smooth metric $g(t)$, $t \in] 0, T]$, the operator $\Delta_{g(t)}$ depends smoothly on $t$. The main obstacle here is that we are not sure of the existence of a complete orthonormal system of eigenfunctions that depend smoothly on $t$. For this purpose, we consider the particular case where $g(t)$ is analytic in $t \in] 0, T\left[\right.$ such that $d v_{g(t)}=d v_{g(0)}$ for all $t \in] 0, T]$.

Remark 3.1. The use of the interval $] 0, T[$ instead of the interval $]-T, T[$ is due to the fact that in Theorem 3.3, $t$ must be positive.

We have the following: 
Definition 3.2 (T. Kato [6] page 375). Let $X$ and $Y$ be two vector spaces, and let $\mathcal{C}(X, Y)$ denote the set of all closed operators from $M$ to $N$. A family $T(x) \in \mathcal{C}(X, Y)$ defined for $x$ in a domain $D_{\mathbb{C}}$ of the complex plane, is said to be holomorphic of type (A) in the sense of Kato if

(i) $D(T(x))=D_{T}$ is independent of $x$, and

(ii) $T(x) u$ is holomorphic for $x \in D_{\mathbb{C}}$ for every $u \in D_{T}$.

Let $g(t)$ be an analytic metric in $t$. Then it can be expressed as follows

$$
g(t)=\sum_{i=0}^{\infty} a_{i} t^{i}
$$

We expand $g$ on $\mathbb{C}$ via the formula

$$
g(z)=\sum_{i=0}^{\infty} a_{i} z^{i} .
$$

We associate to $g(z)$ the operator $\Delta_{g(z)}$ of $C^{\infty}(M, \mathbb{C})$ defined by

$$
\Delta_{g(z)} f=g^{i j}(z)\left[\frac{\partial^{2}}{\partial x^{i} \partial x^{j}} f-\Gamma_{i j}^{k} \frac{\partial}{\partial x^{k}} f\right],
$$

where $\left(g^{i j}(z)\right)_{i, j}=g(z)^{-1}$ and

$$
\Gamma_{i j}^{k}(z)=g^{k l}(z)\left(\frac{\partial}{\partial x^{i}} g_{l j}(z)+\frac{\partial}{\partial x^{j}} g_{l i}-\frac{\partial}{\partial x_{l}} g_{i j}(z)\right) .
$$
(A).

Then by P. Bérard [1], the family $\Delta_{g(t)}$ is an holomorphic family of type

By a theorem due to T. Kato ([6], Theorem 8.9, page 392), there exist a sequence of scalar-valued functions $\lambda_{i}(t)$ and a sequence of vector-valued functions $\varphi_{i}(t)$ all holomorphic in $] 0, T$ [ such that the $\lambda_{i}(t)$ represent all the eigenvalues of $\Delta_{g(t)}$, and the $\varphi_{i}(t)$ form a complete orthonormal basis of associated eigenvectors of $\Delta_{g(t)}$. Hence we can formulate the following statement.

Theorem 3.3. Let $(M, g(t))$ be a family of Riemannian manifolds such that $g(t)$ is analytic in $t \in] 0, T\left[\right.$. And let $\left\{\phi_{i}(t)\right\}_{i \geq 0}$ be a complete orthonormal eigenfunctions of $\Delta_{g(t)}$, associated to the eigenfunctions $\left\{\lambda_{i}(t)\right\}_{i \geq 0}$, all analytic in $t \in] 0, T[$. Then the map

$$
\begin{gathered}
\left.\Phi_{t}:(M, g(t)) \rightarrow l^{2}, \quad t \in\right] 0, T[ \\
x \mapsto \sqrt{2}(4 \pi)^{\frac{n}{4}} t^{(n+2) / 4}\left\{e^{-\frac{\lambda_{i}(t) t}{2}} \varphi_{i}(t, x)\right\}_{i \geq 1}
\end{gathered}
$$

is an embedding for all $t \in] 0, T]$, and

$$
\Phi_{t}^{*}(V, V)=g(t)(V, V)+\frac{t}{3}\left(\frac{1}{2} \operatorname{Scal}_{g(t)} . g(t)-\operatorname{Ric}_{g(t)}\right)+O\left(t^{2}\right) \text { when } t \rightarrow 0_{+}
$$

where $S_{c a l}(t)$ and $R_{i c} c_{g(t)}$ are respectively the scalar curvature and the Ricci curvature tensor of the metric $g(t)$. 
Proof. The proof of this theorem is analogous to the proof of Theorem 1.1 in [3]. It is sufficient to consider the heat kernel $k_{s}$ of $(M, g(s))$ for $s$ fixed :

$$
k_{s}(t, x, y)=\sum_{i \in \mathbb{N}} e^{-\lambda_{i}(s) t} \varphi_{i}(s, x) \varphi_{i}(s, y), \quad t>0, x, y \in M .
$$

Then $\Phi_{t}$ is an embedding of $(M, g(t))$ in $l^{2}$, continuous in $t$, and, for all $\left.t \in\right] 0, T$, and all $x \in M$,

$$
\left\|\Phi_{t}(x)\right\|_{l^{2}}^{2}=\sum_{i>0} e^{-\lambda_{i}(t) t} \varphi_{i}^{2}(t, x)=k_{t}(t, x, x) .
$$

Acknowledgments. I would like to thank the Lebanese National Council for Scientific Research (CNRS-Lebanon) for financing my doctoral studies at Université de Grenoble, and I would like to thank professor Pierre Bérard, and Gérard Besson for help, and for many useful discussions.

\section{References}

[1] P. Bérard, Sur un lemme de perturbation, to appear.

[2] _ Volume des ensembles nodaux des fonctions propres du laplacien, BonySjöstrand-Meyer seminar, 1984-1985, pages Exp. No 14, 10. École polytech., Palaiseau, 1985.

[3] P. Bérard, G. Besson, and S. Gallot, Embedding Riemannian manifolds by their heat kernel, Geom. Funct Anal. 4 (1994), no. 4, 373-398.

[4] R. R. Coifman and Stéphane Lafon, Diffusion maps, Appl. Comput. Harmon. Anal. 21 (2006), no. 1, 5-30.

[5] M. P. do Carmo, Riemannian Geometry, Birkhäuser, 1992.

[6] T. Kato, Perturbation Theory for Linear Operators, Second edition. Grundlehren der Mathematischen Wissenschaften, Band 132. Springer-Verlag, Berlin-New York, 1976.

[7] J. Korevaar, Tauberian Theory, Springer-Verlag, Berlin, 2004.

[8] C. Morlet, Le lemme de Thom et les théorèmes de plongement de Whitney. II, Quelques ouverts fondamentaux des espaces d'applications. Séminaire Henri Cartan, 1961/62, Exp. 5, page 6. Secrétariat mathématique, Paris, 1961/1962.

[9] F. Rellich, Perturbation Theory of Eigenvalue Problems, Assisted by J. Berkowitz. With a preface by Jacob T. Schwartz. Gordon and Breach Science Publishers, New York, 1969.

Université DE GREnoble 1

INSTITUT FOURIER

LABORATOIRE DE MATHÉmatiques associé AU CNRS

B. P. 74, 38042 Saint Martin D'Hères Cedex France

E-mail address: Hiba.Abdallah@ujf-grenoble.fr 